Review Article

\title{
Faecalibacterium prausnitzii: A Next-Generation Probiotic in Gut Disease Improvement
}

\author{
Xiaoya He, ${ }^{1}$ Shuyang Zhao, ${ }^{2}$ and Yan Li ${ }^{1}$ \\ ${ }^{1}$ State Key Laboratory of Oral Diseases, National Clinical Research Center for Oral Diseases, West China Hospital of Stomatology, \\ Sichuan University, No. 14, Section 3, Renmin South Road, Chengdu 610041, China \\ ${ }^{2}$ Queen Mary School of Medical College, Jiangxi Medical College, Qianhu Campus, Nanchang University, No. 1290 Xuefu Street, \\ Jiangxi 330031, China \\ Correspondence should be addressed to Yan Li; feifeiliyan@163.com
}

Received 20 November 2020; Revised 9 January 2021; Accepted 24 February 2021; Published 5 March 2021

Academic Editor: Tingtao Chen

Copyright $\odot 2021$ Xiaoya He et al. This is an open access article distributed under the Creative Commons Attribution License, which permits unrestricted use, distribution, and reproduction in any medium, provided the original work is properly cited.

\begin{abstract}
The researchers are paying more attention to the role of gut commensal bacteria in health development beyond the classical pathogens. It has been widely demonstrated that dysbiosis, which means the alternations of the gut microbial structure, is closely associated with development of intestinal chronic inflammation-related diseases such as inflammatory bowel disease (IBD), and even infectious diseases including bacterial and viral infection. Thus, for reshaping ecological balance, a growing body of the literatures have proposed numerous strategies to modulate the structure of the gut microbiota, which provide more revelation for amelioration of these inflammation or infection-related diseases. While the ameliorative effects of traditional probiotics seem negligeable, emerging next generation probiotics (NGPs) start to receive great attention as new preventive and therapeutic tools. Encouragingly, within the last decade, the intestinal symbiotic bacterium Faecalibacterium prausnitzii has emerged as the "sentinel of the gut," with multifunction of anti-inflammation, gut barrier enhancement, and butyrate production. A lower abundance of $F$. prausnitzii has been shown in IBD, Clostridium difficile infection (CDI), and virus infection such as COVID-19. It is reported that intervention with higher richness of $F$. prausnitzii through dietary modulation, fecal microbiota transplantation, or culture strategy can protect the mice or the subjects from inflammatory diseases. Therefore, F. prausnitzii may have potential ability to reduce microbial translocation and inflammation, preventing occurrences of gastrointestinal comorbidities especially in COVID-19 patients.
\end{abstract}

\section{Introduction}

Probiotics are demonstrated to have potential anti-inflammatory and antiviral effects $[1,2]$. The safety and clinical efficacy in reducing the severity and duration of upper respiratory tract infections of probiotics have been demonstrated [3]. An earlier review [4] confirmed the prophylactic and therapeutic effects of several lactic acid-producing bacteria strains on viral infection, which are reported to decrease titres of Ebola and cytomegalovirus and reduce respiratory and intestinal inflammation accordingly. Recently, Eguchi et al. evaluated the ability of Lactobacillus gasseri against respiratory syncytial virus (RSV) in a mice model [5]. The decreasing RSV titre and the diminishing expression of proinflammatory cytokines in the lung were significantly observed while interferons and interferon-stimulated genes were increased after the treatment. In addition, by summarizing 15 studies of Lactobacillus rhamnosus GG in the treatment of acute diarrhea, Szajewska and Kołodziej [6] concluded that Lactobacillus rhamnosus $G G$ could reduce the severity of purging and the duration of diarrhea by about 1 day, and the most effective dose was more than $10^{10} \mathrm{CFU}$. According to a meta-analysis, a lower dose Lactobacillus reuteri was also reported to be effective in reducing the duration of diarrhea by approximately 1 day [7].

Although most traditional and widely used probiotics (e.g., Bifidobacterium spp. and Lactobacillus spp.) are safe, their effect on disease improvement is uncertain. Moreover, traditional probiotics are not disease-specific. Based on these situations, there is an urgent need for identification and 
characterization of novel and disease-specific next generation probiotics (NGP). As one of the most common microbial species in the colon of healthy adults, Faecalibacterium prausnitzii (F. prausnitzii) constitutes over 5 percent of the overall total bacterial population [8]. The members of the genus Faecalibacterium are deemed symbiotic microorganisms, omnipresent in human and animal gastrointestinal tracts (GIT) [9]. Alterations in the abundance of $F$. prausnitzii have been commonly identified to be related to a number of human intestinal and metabolic diseases [10]. Therefore, due to the pervasiveness and immunomodulator, F. prausnitzii is not only an important predictor but also an influential contributor to intestinal health as well as the maintenance of gut homeostasis.

Studies have shown a certain correlation between the low abundance of $F$. prausnitzii and the increased incidence of inflammatory metabolic diseases such as inflammatory bowel disease [11], Crohn's disease [12], colitis [13] and some infectious diseases such as Clostridium difficile infection [14], human immunodeficiency virus (HIV) [15], and hepatitis B virus (HBV) [16]. On the other hand, supplementation with $F$. prausnitzii may contribute to the amelioration of specific metabolic disorders and inflammatory diseases [17-19].

Given the above, it is fair to speculate that these antiinflammatory and antiviral effects can well lead to the prevention and/or relief of COVID-19-related symptoms, at least partially or in combination with other medicines. Thus, this raises a possibility that $F$. prausnitzii might be a new candidate probiotic which can be used in COVID-19 patients. Herein, we discuss recent advances in the understanding of the protective effects and mechanisms on infectious diseases of $F$. prausnitzii and its potential relevance in COVID-19 infection.

\section{The Bionomics of $\boldsymbol{F}$. prausnitzii}

F. prausnitzii is one of the most abundant and widely distributed bacterial species inhabiting the human intestine, which has been consistently described as one of the most important butyrate producers found in the intestine [20]. Taxonomically, F. prausnitzii belongs to the Firmicutes phylum, the Clostridia class, and the Ruminococcaceae family, and the species is currently the only representative characterized within the genus [9]. Metabolically, as an anaerobe, F. prausnitzii, a non-spore-forming and nonmotile rod that is Gram-positive, is exceedingly oxygensensitive [9]. It is difficult to survive even in an anaerobic environment, but adding riboflavin, cysteine or glutathione to the culture medium can improve its survival rate in a microaerobic environment [21]. F. prausnitzii can be divided into two lineages, line I and line II, and the differences in their physiological functions are still unclear [21].

A variety of monosaccharides can be used by bacteria as their energy sources, while the use of more complex carbohydrates varies from strain to strain. The nutrients can be obtained from the host or derived from other gut microbes cross-feeding. F. prausnitzii can use fructose, oligofructose, starch and inulin, but not arabinose, melibiose, raffinose, rhamnose, ribose, and xylose. Acetic acid can stimulate its growth and produce carbon dioxide, but not hydrogen [9]. The major fermentation products from glucoses and acetate by $F$. prausnitzii are formate, D-lactate, and butyrate [9].

The proportion of $F$. prausnitzii in gut microbiota is flexible affected by the colon physiological environment such as the $\mathrm{pH}$, oxygen concentration, and cholate $[21,22]$. Moreover, both improper diet and smoking will lead to a decreased count of $F$. prausnitzii [23]. In addition, the use of certain drugs can also modulate the abundance of $F$. prausnitzii in the intestine. For example, taking rifaximin can increase the level of $F$. prausnitzii [24], while the bacterium in the stool of Crohn's disease patients is reduced by taking infliximab and high doses of cortisol [25]. Studies have reported that butyrate produced by $F$. prausnitzii has a significant protective effect on enteritis [25]. As butyrate-producing bacteria, F. prausnitzii acts on the immune system and has anticancer effects $[26,27]$. It can also improve the intestinal barrier, insulin sensitivity, and oxidative stress tolerance and reduce visceral sensitivity [28, 29].

Moreover, as one of the most abundant gut commensal bacteria, F. prausnitzii has the double effect of competitively inhibiting pathogenic bacteria and increasing the colonization of nonpathogenic bacteria [30], which could maintain a normal proportion of the gut microbiota. When F. prausnitzii is cocultured with Bacteroides thetaiotaomicron (B. thetaiotaomicron), which can also metabolize apple pectin, F. prausnitzii can produce more butyric acid than it alone [31]. This indicates that $F$. prausnitzii may rely on other gut microbiota including $B$. thetaiotaomicron for cross-feeding. Some studies found that the colonization of $F$. prausnitzii requires B. thetaiotaomicron and Escherichia coli (E. coli) to be preexisted in the intestine, which could prepare suitable conditions for $F$. prausnitzii by reducing redox potential and altering the composition of nutrients [32, 33]. In addition, F. prausnitzii and normal intestinal microecology can effectively prevent the proliferation of intestinal pathogenic bacteria such as Escherichia coli, Clostridium, and Shigella, which reduce the possibility of intestinal epithelium injury, thereby avoiding the activation of intestinal immune cells and the release of inflammatory factors [34].

Therefore, $F$. prausnitzii is a probiotic with an important protective effect on the human intestine and its reduction will lead to weakened intestinal anti-inflammatory and immune regulation capabilities.

Some characteristics of $F$. prausnitzii such as the absence of adhesion of epithelial cells [35], plasmids, antimicrobial $[19,36]$ and hemolytic activity, and the presence of DNAse activity [19] have been known to date. In addition, only the reference strain F. prausnitzii A2-165 [37] and the biofilm forming strain HTF-F [38] have been examined in vitro and in vivo for their beneficial anti-inflammatory effects. Some evidence points to this symbiotic intestinal bacterium, associated with intestinal barrier integrity and inflammation regulation, as an emerging "gatekeeper of the gut." 


\section{Multiskilled Commensal Bacterium F. prausnitzii}

As a major member of the human microbiome, F. prausnitzii is a multiskilled commensal organism. It is distributed widely in the mammalian digestive tract. This bacterium has a variety of biological functions, such as regulating the immune response, suppressing inflammation, and promoting the integrity of the intestinal barrier.

3.1. Anti-inflammatory Effects. F. prausnitzii is a commensal bacterium with anti-inflammatory property, as demonstrated in a clinical trial [32]. Various studies have demonstrated decreasing abundance of $F$. prausnitzii in the gut could reduce protection against inflammatory interactions. This defensive mechanism possibly involves stimulating active molecules to secrete anti-inflammatory while inhibiting the secretion of proinflammatory cytokines. $F$. prausnitzii secretes anti-inflammatory molecules which can block the IL- $1 \beta$-induced NF- $\kappa \beta$ signaling pathway, thereby reducing the production of interleukin IL-8 secreted by intestinal epithelial cells [39]. Additionally, F. prausnitzii can promote the secretion of IL-10 through peripheral blood monocytes, dendritic cells (DCs), and macrophages [37, 40] and consequently inhabit the synthesis of proinflammatory cytokines such as IFN- $\gamma$, TNF- $\alpha$, IL-6, and IL-12 [41]. Through these mechanisms, the anti-inflammatory effect of $F$. prausnitzii in colitis may be realized, and through its antiinflammatory properties, this bacterium may promote intestinal immune homeostasis.

3.2. Enhancement of Gut Barrier Function. Another key to intestinal development and maturity is the establishment of the integrity of the intestinal mucosa, which is not only essential for the absorption of nutrients, but also necessary for preventing bacteria and food antigens from entering the underlying tissues [42]. The metabolites released by F. prausnitzii have been shown to reduce the severity of inflammation by improving the function of the intestinal barrier and affecting paracellular permeability [43, 44]. Rossi et al. reported that the cell-free supernatant of $F$. prausnitzii can enhance the intestinal mucus barrier function by affecting the permeability of epithelial cells [40]. The improvement in permeability of $F$. prausnitzii appears to be related to the expression of certain tightly bound proteins [43]. It was investigated that $F$. prausnitzii could increase the levels of tight junction proteins occludin and E-cadherin and decrease colonic permeability, alleviating inflammation both in vitro and in vivo [45]. Moreover, F. prausnitzii may also help to maintain sufficient proportions of various cell types of secretory linage in the intestinal epithelium via the mucus pathway and O-glycan mucus formation [31].

3.3. Effects of Metabolites. Although we have confirmed the anti-inflammatory property of $F$. prausnitzii and its supernatant, the exact active substance and its mechanism have not been fully elucidated due to its complex composition. As an acetate consumer, $F$. prausnitzii has capacity to generate anti-inflammatory molecules such as butyrate and salicylic acids [32]. A growing body of the literature has reported that the main metabolites of $F$. prausnitzii, butyrate, play an important role in its antiinflammatory activity. Butyrate is a short-chain fatty acid (SCFA) produced by intestinal microorganisms fermenting dietary fiber [46]. Moreover, F. prausnitzii has been consistently regarded as one of the main butyrate producers found in the intestine [47]. Butyrate provides energy (5-15\% of the total calories) to the host and regulates the immune system, thereby protecting the host from pathogens [48].

Butyrate is secreted by intestinal microbiota and plays a significant role in intestinal physiology and body function. It is of great importance to prevent the invasion of pathogens, regulate the immune system, and reduce cancer progression [49]. As a representative of numerous pathways for electron disposal in the gut microbiota, F. prausnitzii can form butyrate, and its concomitant generation of $\mathrm{NAD}^{+}$and decreased ferredoxin is able to facilitate immune response modulation.

Salicylic acid is another metabolic product with antiinflammatory effects delivered by $F$. prausnitzii [32]. As strong modulators of the inflammatory process, salicylic acid can also block the activation of NF- $\kappa \beta$ to inhibit the production of IL-8 as same as butyrate [50]. In the pharmaceutical industry, it is commonly recognized that salicylic acid can work as the forerunner of 5-aminosalicylic acid (5-ASA), which is a drug prescribed in the management procedure of IBD [24]. It has been documented in vitro that $10 \mathrm{mM}$ of salicylic acid could reduce the level of IL- 8 as well as the concentration found in the colon [32].

In addition, F. prausnitzii is capable of secreting antiinflammatory substances including butyrate and salicylic acid, as previously described. Quévrain et al. reported another anti-inflammatory compound producing by F. prausnitzii called microbial anti-inflammatory molecule (MAM), which can inhibit the trigger of NF- $\kappa \mathrm{B}$ in vitro and vivo as well $[18,34]$. Since MAM operates actively at the center of signaling molecule I $\kappa \mathrm{B}$ kinase $\alpha$ (IKK $\alpha$ ), extra management of signal transduction molecules upstream or downstream of IKK $\alpha$ may significantly enhance its effects. In general, MAM could reach all over the body, and it can play a significant role in the regulation of inflammatory complications at anatomical locations outside of the intestine. The main mechanisms of F. prausnitzii are shown in Figure 1.

\section{The Diseases Related to F. prausnitzii}

As an important part of healthy human gut commensals, F. prausnitzii exerts significant actions on human health. Accumulating studies showed that the dysbiosis caused by the change of the count of $F$. prausnitzii in the intestine was closely related to the onset of some intestinal diseases such as IBD, irritable bowel syndrome (IBS), and colorectal cancer (CRC) $[37,51,52]$. 


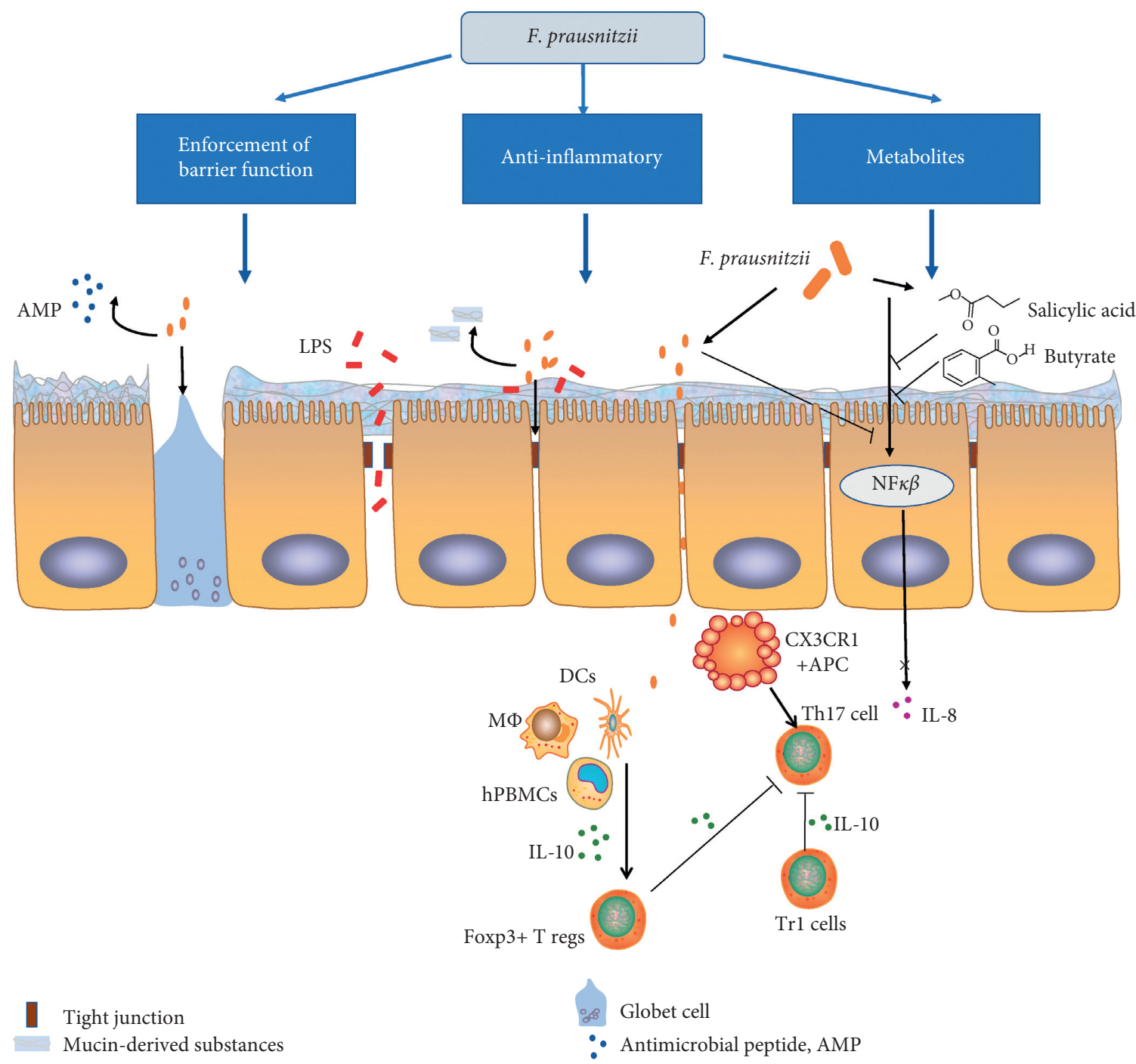

FIGURE 1: Mechanism underlying the anti-inflammation and enhancement of barrier function of Faecalibacterium prausnitzii. The effector molecules of F. prausnitzii encourage secretion of IL-10 from peripheral blood mononuclear cells (PBMCs), dendritic cells (DCs), and macrophages, and thus, proinflammatory cytokines are downregulated. The metabolites from $F$. prausnitzii, butyrate and salicylic acid, can block the secretion of IL- 8 through inhibition of activation NF- $\kappa \beta$ signaling and inflammation. The metabolites and effector molecules of F. prausnitzii can enhance the barrier function through providing growth factors from mucin degradation. F. prausnitzii could interact with the host by strengthening the intestinal barrier or modulating mucin turnover and immune responses.

4.1. IBD. Inflammatory bowel disease (IBD), whose two major forms are ulcerative colitis (UC) and Crohn's disease (CD) [53], is a chronic inflammation of the intestine induced by immune response under environmental conditions such as genetic susceptibility, diet, and antibiotic use. In the past few decades, plenty of clinical research data has shown that the composition and diversity of microbiota is modified in patients with IBD. Compared to that in healthy people, an increasing abundance of Proteobacteria was observed in the fecal microbiota of patients with active CD and UC, while lower fecal counts of Firmicutes were detected [37]. F. prausnitzii, as the most abundant bacteria in human intestine, possesses a small amount in CD and UC patients [54]. Machiels et al. found that there was a significant inverse association between the count of F. prausnitzii and disease activity in UC patients, even with the inactive disease [55]. Moreover, regarding the metabolites, decreasing short-chain fatty acids were observed in patients with UC [55]. Recently, Zhao et al. performed a systematic review and meta-analysis involving patients with UC and CD [56]. They found that both CD and UC patients had a lower abundance of F. prausnitzii than the healthy controls and a lower count of $F$. prausnitzii was detected in patients with active IBD in contrast with those in remission. In addition, it was reported 
that patients receiving infliximab, a TNF- $\alpha$ blocker, showed an increase in F. prausnitzii population [57], which suggested a relationship in the pathomechanisms of IBD.

4.2. CDI. In addition to gut diseases, recent studies have shown the potential relevance between $F$. prausnitzii and infectious diseases such as CDI, HIV, and HBV. This will undoubtedly be a hotspot on F. prausnitzii worth studying in the future.

A study reported that patients with CDI had significantly fewer members of $F$. prausnitzii in their fecal microbiota than the healthy group [58]. Demirci et al. found that the amount of $F$. prausnitzii was reduced in patients with allergic diseases, which might suppress inflammation by decreasing proinflammatory cytokines such as IL-12 and increasing anti-inflammatory cytokine IL-10 [59]. Roychowdhury et al. revealed that supplementation with anti-inflammatory butyrate-producing commensal bacteria and prebiotic might help to promote innate immune responses and minimize bacterial burden and adverse effects during a course of antibiotic and Clostridium difficile exposure [60]. Moreover, a study observed increased $F$. prausnitzii in a cured recurrent CDI patient who had received fecal microbiota transplantation (FMT) 4.5 years ago.

Le Bastard et al. [61] discovered that ampicillin resulted in a sharp drop in bacterial species richness and diversity along with a fall in the percentage of $F$. prausnitzii. In mice receiving FMT, dysbiosis was immediately reversed with a significant increase in F. prausnitzii.

4.3. Virus-Infected Gut Dysfunction. Furthermore, Lu et al. found that gastrointestinal microbiota changes were linked to $\mathrm{CD} 4^{+} \mathrm{T}$-cell counts and immune activation in those with HIV [14]. In that study, F. prausnitzii is overrepresented in $\mathrm{HIV}$-infected individuals who are immunological ART nonresponders or untreated compared to those immunological ART responders. Similarly, another study revealed that F. prausnitzii was depleted in HIV-positive persons on long-term ART compared to HIV-negative and the amount of $F$. prausnitzii has a negative correlation with gut dysfunction [15]. It has also been observed that the count of F. prausnitzii in asymptomatic carriers showed significant variation, and the variation range was considerably higher in patients with chronic hepatitis B and those with decompensated HBV cirrhosis in comparison with healthy controls [16]. These findings have revealed the potential connection between F. prausnitzii and viral infectious diseases, suggesting the possibility of $F$. prausnitzii as a targeted probiotic in the treatment of viral infectious diseases.

\section{Strategies to Modulate the Abundance of F. prausnitzii}

Due to the fact that all the $F$. prausnitzii strains from feces of healthy individuals showed positive anti-inflammation [62], it could be a promising target for therapeutic purpose. However, the production of medical supplements using obligate anaerobes, such as F. prausnitzii, is certainly a major challenge owing to the requirements for anaerobic conditions and mass production. Herein, we propose strategies to increase the abundance of $F$. prausnitzii from three perspectives.

5.1. Dietary Interventions Modulate F. prausnitzii. The structure of microbial communities of human beings depends, to a great extent, on the dietary factor, for bacterial composition in the gut is closely related to the available nutritional compounds [62]. The intake of the typical westernized diet which means a large amount of animal meat, sugar, animal fat, processed foods, and low fiber diet could reduce $F$. prausnitzii, whereas a high fiber diet with less meat can increase F. prausnitzii [63]. Hence, the abundance of $F$. prausnitzii can be regulated through the consumption of prebiotics and/or formulations. Treatment with inulin-type fructans and fructo-oligosaccharides has been demonstrated to increase the level of F. prausnitzii compared to placebo (maltodextrin) in patients [64]. Supplementation of prebiotic inulin-oligofructose also led to an increase of F. prausnitzii in healthy individuals [65]. An increase of $F$. prausnitzii was reported for red wine intake compared to baseline in male metabolic syndrome patients and healthy individuals [66]. Another study found that healthy men who consumed polydextrose or soluble corn fiber supplements had more F. prausnitzii than men who did not take fiber supplements, suggesting that this might be potential prebiotics [67]. In a research on the effects of a lowenergy diet with prebiotic properties for patients with type 2 diabetes, $F$. prausnitzii increased by $34 \%$ compared to a placebo diet [68].

F. prausnitzii is reported to consume various diet including polysaccharides, such as the prebiotic inulin, arabinoxylans, resistant starch, fructan supplement, and ectins $[63,69]$. As the most important modulators of gut microbiota, polysaccharides are generally consumed in the food because of their relative security, availability, and low price. A study demonstrated that increased consumption of polysaccharides had the potential to give advantage to individuals with a typical western-style diet, on condition that they take in enough dietary fiber [70]. As shown in several meta-analyses, the increased intake of dietary fiber greatly reduces the mortality risk [71].

5.2. Fecal Microbiota Transplantation. In recent years, there has been considerable interest in FMT, which is implemented by transferring the microbiota from healthy donors to people suffering from dysbiosis to restore eubiotic state. In an earlier study, van Nood et al. demonstrated that FMT could heal recurrent CDI, targeting the gut microbiota which can exert profound influence on the host metabolism [72]. Furthermore, researchers found that infusion of donor feces was significantly more effective for the treatment of recurrent CDI than the use of antibiotics. Moreover, in order to treat Crohn's disease and ulcerative colitis, Cui et al. proposed a step-up FMT strategy consisting of a FMT, then by further FMT steps or standard IBD prescriptions depending upon the patient's therapeutic response [73]. It is known that FMT can influence the growth of 
Bacteroidetes and Firmicutes, especially F. prausnitzii [74]. A recent study investigates the safety and effectiveness of FMT in patients with mild or moderate UC by giving 47 patients treatments of fresh FMT. It shows that FMT resulted in clinical remission in patients with mild to moderate UC and that the remission may be associated with significantly increased levels of F. prausnitzii [75]. Sarrabayrouse et al. investigate changes in recipient intestinal mucosa upon contact with a fecal suspension (FS) obtained from a healthy donor by using a human explant tissue model and an in vivo mouse model. Interestingly, it shows that tissues with a low microbial load and a higher relative abundance of Firmicutes were more susceptible to FMT [76]. These studies suggest that F. prausnitzii can be a diagnostic and therapeutic candidate for the use of FMT in UC.

However, beneficial effects of FMT can be affected by dietary and host immune factors. The microbial structure of a healthy individual must be taken into account in time and in accordance with dietary, immune, and aging influences. In addition, the potential risk of the transmission of obesity and metabolic syndrome associated flora ought not to be ignored. FMT from the obese ones caused an increase in adiposity in mice, indicating the potential risk of transmission of some diseases associated flora [77]. Therefore, FMT needs further assessment.

5.3. Cultivate F. prausnitzii In Vitro. In addition to fecal microbiota transplantation and dietary regulation, how to cultivate $F$. prausnitzii in vitro is of vital importance. At present, the research on the isolation of F. prausnitzii and the exploration of the relationship between $F$. prausnitzii and diseases from the strain level are still in the preliminary stage at home and abroad. Therefore, the isolation and identification of F. prausnitzii and the screening of excellent strains that are correlated with human health and have strong biological activity have become an important prerequisite for further indepth research on it. F. prausnitzii is extremely oxygen-sensitive, which may lose validity when exposed to the air for 2 minutes, and it is difficult to cultivate even in an anaerobic environment [9]. Adding riboflavin and cysteine or glutathione to the medium can make it grow in low oxygen environment [78]. In order to adapt to the micro-oxygen environment in the intestine, F. prausnitzii uses flavin and thiols as shuttle carriers inside and outside the cell to transfer electrons to oxygen and protect itself from oxidative stress [78]. Khan et al. found that the obligate anaerobic F prausnitzii can be kept alive at ambient air for $24 \mathrm{~h}$ in a growth medium formulated with the antioxidants cysteine and riboflavin plus the cryoprotectant inulin [79]. It suggests that we can improve the growth of F. prausnitzii in vitro by changing the composition of the medium.

\section{Potential Clinical Applications of F. prausnitzii in Diseases, Even in COVID- 19 Infection}

Since $F$. prausnitzii is extremely sensitive to changes in the intestinal environment, fecal- or mucosal-related $F$. prausnitzii can be regarded as a potential biomarker for diagnosis of intestinal diseases. However, a single bacterial species cannot be a universal biomarker for all types of diseases. Lopez-Siles proposed that the F-E index obtained by combining the abundance of $F$. prausnitzii and E. coli can be a better indicator than the single specie [80], which could discriminate between CD, IBS, and CRC $[25,52,80]$, also distinguish CRC patients from other intestinal diseases. Since IBD is a chronic disease, many researchers paid their attention to the use of biomarkers to predict its prognosis. The lower CD activity index, C-reactive protein levels, and erythrocyte sedimentation rate have been demonstrated to be related to higher $F$. prausnitzii counts in feces [12]. Furthermore, disease remission could recover the abundance of $F$. prausnitzii in feces $[13,25]$. However, the current research studies on the characteristics and functions of this bacteria are not enough intensive and extensive. There is a need for more in-depth study on the functional activity of F. prausnitzii and its potential as a biomarker.

In addition, as a treatment strategy, transplantation of F. prausnitzii has been widely used in dysbiosis of the intestinal flora that is associated with the inflammation, autoimmune disease, and infectious diseases. Butyrate-producing bacteria have been demonstrated to prevent translocation of endotoxic, which is a compound produced by the gut microbiota and has been reported to drive insulin resistance [81]. Sokol et al. [13] designed an in vitro experiment to prove that human immune cells with $F$. prausnitzii can show a potential anti-inflammatory response in the gut. At the same time, they revealed that the transplantation of $F$. prausnitzii in mice could shield the gut epithelium from destruction and inhibit gut inflammation induced by experimental reagents. Additionally, several research studies have demonstrated that oral F. prausnitzii has an anti-inflammatory effect in IBD mice models [82, 83]. Overall, transplantation of gut microbiota particularly F. prausnitzii from a healthy individual to subjects with metabolic syndrome or intestinal inflammation could modulate dysbiosis and inhibit downstream proinflammatory response.

Furthermore, respiratory viral infections have been reported to be associated with altered gut microbial structure, which predispose patients to secondary bacterial infections [84]. Probiotics may be an effective adjuvant strategy for the treatment and prophylaxis of viral infections including COVID-19. Numerous experts and scholars have proposed the use of probiotics to participate in the treatment of COVID-19 $[85,86]$, so it is important to screen out new probiotics. Moreover, according to a recent science blog by the IASPP (International Scientific Association for Probiotics and Prebiotics), numerous researchers around the world are studying the susceptibility of the microbiome to COVID-19 and assessing the ability of various probiotic strains to reduce viral load through multiple mechanisms of action.

Researchers performed transcriptome sequencing on the bronchoalveolar lavage fluid of COVID-19 patients, and the results showed that the microbiota was dominated by pathogens or oral and upper respiratory commensal bacteria [87]. Furthermore, it has been demonstrated comorbidities generally associated with severe COVID-19 are closely related to alterations in bacteria taxa from the phyla Bacteroidetes and Firmicutes $[88,89]$, which were reported to regulate ACE2 expression in rodents. 
To the best of our knowledge, ACE2 is known as the receptor for SARS-CoV-2 to enter the host, which is highly expressed in both the respiratory and gastrointestinal tract [90]. In addition, it plays a role in controlling intestinal inflammation and maintaining intestinal microbial ecology [91]. Interestingly, Firmicutes species seemed to have diverse roles in regulating ACE2 expression in the colon of mice models [92]. Moreover, Zuo et al. found that there was an inverse correlation between abundance of $F$. prausnitzii and COVID-19 severity [93]. In this study, F. prausnitzii was discovered to be one of the top bacterial species which show a negative correlation with COVID-19 severity.

Clinical data showed that older patients and those with underlying chronic diseases related to inflammation (such as hypertension, obesity, diabetes, and coronary artery disease) had higher SARS-CoV-2 mortality and morbidity [94, 95]. Interestingly, the abundance of $F$. prausnitzii was reported to be lower in these subjects compared with healthy individuals [96-98]. Hence, it seems reasonable that F. prausnitzii can be an add-on therapy for the management of COVID-19.

The possible role of $F$. prausnitzii abundance in COVID19 infection in terms of gut integrity and inflammation needs to be further elucidated. Its potential prognostic and therapeutic value in SARS-CoV-2 infections is waiting to be explored.

\section{Conflicts of Interest}

The authors declare that there are no conflicts of interest regarding the publication of this paper.

\section{Acknowledgments}

This work was supported by grants from the National Natural Science Foundation of China (81771085) and Key Projects of Sichuan Provincial Department of Science and Technology (2020YFSY0008).

\section{References}

[1] D. E. Kiousi, "Probiotics in extraintestinal diseases: current trends and new directions," Nutrients, vol. 11, no. 4, p. 788, 2019.

[2] B. Strasser et al., "Probiotic supplements beneficially affect tryptophan-kynurenine metabolism and reduce the incidence of upper respiratory tract infections in trained athletes: a randomized, double-blinded, placebo-controlled trial," $\mathrm{Nu}$ trients, vol. 8, no. 11, 2016.

[3] L. Tapiovaara, L. Lehtoranta, T. Poussa, H. Mäkivuokko, R. Korpela, and A. Pitkäranta, "Absence of adverse events in healthy individuals using probiotics-analysis of six randomised studies by one study group," Beneficial Microbes, vol. 7, no. 2, pp. 161-169, 2016.

[4] O. Kanauchi, A. Andoh, S. AbuBakar, and N. Yamamoto, "Probiotics and paraprobiotics in viral infection: clinical application and effects on the innate and acquired immune systems," Current Pharmaceutical Design, vol. 24, no. 6, pp. 710-717, 2018.

[5] K. Eguchi, N. Fujitani, H. Nakagawa, and T. Miyazaki, "Prevention of respiratory syncytial virus infection with probiotic lactic acid bacterium Lactobacillus gasseri SBT2055," Scientific Reports, vol. 9, no. 1, p. 4812, 2019.

[6] H. Szajewska and M. Kołodziej, "Systematic review with metaanalysis:Lactobacillus rhamnosusGG in the prevention of antibiotic-associated diarrhoea in children and adults," Alimentary Pharmacology \& Therapeutics, vol. 42, no. 10, pp. 1149-1157, 2015.

[7] M. Urbańska, D. Gieruszczak-Białek, and H. Szajewska, "Systematic review with meta-analysis: Lactobacillus reuteri DSM 17938 for diarrhoeal diseases in children," Alimentary Pharmacology \& Therapeutics, vol. 43, no. 10, pp. 1025-1034, 2016.

[8] Y. Cao, J. Shen, and Z. H. Ran, "Association between Faecalibacterium prausnitzii reduction and inflammatory bowel disease: a meta-analysis and systematic review of the literature," Gastroenterology Research and Practice, vol. 2014, Article ID 872725, 2014.

[9] S. H. Duncan, "Growth requirements and fermentation products of Fusobacterium prausnitzii, and a proposal to reclassify it as Faecalibacterium prausnitzii gen. nov., comb. nov," International Journal of Systematic and Evolutionary Microbiology, vol. 52, no. Pt 6, pp. 2141-2146, 2002.

[10] C. V. Ferreira-Halder, A. V. d. S. Faria, and S. S. Andrade, "Action and function of Faecalibacterium prausnitzii in health and disease," Best Practice \& Research Clinical Gastroenterology, vol. 31, no. 6, pp. 643-648, 2017.

[11] M. Lopez-Siles, M. Martinez-Medina, C. Abellà et al., "Mucosa-associated Faecalibacterium prausnitzii phylotype richness is reduced in patients with inflammatory bowel disease," Applied and Environmental Microbiology, vol. 81, no. 21, pp. 7582-7592, 2015.

[12] T. Fujimoto, H. Imaeda, K. Takahashi et al., "Decreased abundance of Faecalibacterium prausnitzii in the gut microbiota of crohn's disease," Journal of Gastroenterology and Hepatology, vol. 28, no. 4, pp. 613-619, 2013.

[13] H. Sokol, P. Seksik, J. P. Furet et al., "Low counts of Faecalibacterium prausnitzii in colitis microbiota," Inflammatory Bowel Diseases, vol. 15, no. 8, pp. 1183-1189, 2009.

[14] W. Lu, "Association between gut microbiota and CD4 recovery in HIV-1 infected patients," Frontiers in Microbiology, vol. 9, p. 1451, 2018.

[15] S. Pinto-Cardoso, "Fecal bacterial communities in treated HIV infected individuals on two antiretroviral regimens," Scientific Reports, vol. 7, p. 43741, 2017.

[16] H. Lu, Z. Wu, W. Xu, J. Yang, Y. Chen, and L. Li, "Intestinal microbiota was assessed in cirrhotic patients with hepatitis B virus infection," Microbial Ecology, vol. 61, no. 3, pp. 693-703, 2011.

[17] X. Qiu, M. Zhang, X. Yang, N. Hong, and C. Yu, "Faecalibacterium prausnitzii upregulates regulatory $\mathrm{T}$ cells and antiinflammatory cytokines in treating TNBS-induced colitis," Journal of Crohn's and Colitis, vol. 7, no. 11, pp. e558-e568, 2013.

[18] N. M. Breyner, "Microbial anti-inflammatory molecule (MAM) from Faecalibacterium prausnitzii shows a protective effect on DNBS and DSS-induced colitis model in mice through inhibition of NF- $\kappa$ B pathway," Frontiers in Microbiology, vol. 8, p. 114, 2017.

[19] R. Martín, S. Miquel, L. Benevides et al., "Functional characterization of novel Faecalibacterium prausnitzii strains isolated from healthy volunteers: a step forward in the use of F. Prausnitzii as a next-generation probiotic," Frontiers in Microbiology, vol. 8, p. 1226, 2017. 
[20] P. Louis and H. J. Flint, "Diversity, metabolism and microbial ecology of butyrate-producing bacteria from the human large intestine," FEMS Microbiology Letters, vol. 294, no. 1, pp. 1-8, 2009.

[21] M. Lopez-Siles, T. M. Khan, S. H. Duncan, H. J. M. Harmsen, L. J. Garcia-Gil, and H. J. Flint, "Cultured representatives of two major phylo groups of human colonic Faecalibacterium prausnitzii can utilize pectin, uronic acids, and host-derived substrates for growth," Applied and Environmental Microbiology, vol. 78, no. 2, pp. 420-428, 2012.

[22] G. L. Hold, A. Schwiertz, R. I. Aminov, M. Blaut, and H. J. Flint, "Oligonucleotide probes that detect quantitatively significant groups of butyrate-producing bacteria in human feces," Applied and Environmental Microbiology, vol. 69, no. 7, pp. 4320-4324, 2003.

[23] R. F. J. Benus, T. S. van der Werf, G. W. Welling et al., "Association between Faecalibacterium prausnitzii and dietary fibre in colonic fermentation in healthy human subjects," British Journal of Nutrition, vol. 104, no. 5, pp. 693-700, 2010.

[24] Y. Dörffel, A. Swidsinski, V. Loening-Baucke, B. Wiedenmann, and M. Pavel, "Common biostructure of the colonic microbiota in neuroendocrine tumors and crohn's disease and the effect of therapy," Inflammatory Bowel Diseases, vol. 18, no. 9, pp. 1663-1671, 2012.

[25] A. Swidsinski, V. Loening-Baucke, M. Vaneechoutte, and Y. Doerffel, “Active Crohn's disease and ulcerative colitis can be specifically diagnosed and monitored based on the biostructure of the fecal flora," Inflammatory Bowel Diseases, vol. 14, no. 2, pp. 147-161, 2008.

[26] J. Hansen, A. Gulati, and R. B. Sartor, "The role of mucosal immunity and host genetics in defining intestinal commensal bacteria," Current Opinion in Gastroenterology, vol. 26, no. 6, pp. 564-571, 2010.

[27] P. L. Miller and T. L. Carson, "Mechanisms and microbial influences on CTLA-4 and PD-1-based immunotherapy in the treatment of cancer: a narrative review," Gut Pathogens, vol. 12, p. 43, 2020.

[28] Q. Zhai, D. Qu, S. Feng et al., "Oral supplementation of leadintolerant intestinal microbes protects against lead $(\mathrm{Pb})$ toxicity in mice," Frontiers in Microbiology, vol. 10, p. 3161, 2019.

[29] N. Mohebali, "Barrier protection and recovery effects of gut commensal bacteria on differentiated intestinal epithelial cells in vitro," Nutrients, vol. 12, no. 8, 2020.

[30] J. C. Clemente, L. K. Ursell, L. W. Parfrey, and R. Knight, “The impact of the gut microbiota on human health: an integrative view," Cell, vol. 148, no. 6, pp. 1258-1270, 2012.

[31] L. Wrzosek, S. Miquel, M.-L. Noordine et al., "Bacteroides thetaiotaomicron and Faecalibacterium prausnitzii influence the production of mucus glycans and the development of goblet cells in the colonic epithelium of a gnotobiotic model rodent," BMC Biology, vol. 11, no. 1, p. 61, 2013.

[32] S. Miquel, M. Leclerc, R. Martin et al., "Identification of metabolic signatures linked to anti-inflammatory effects of Faecalibacterium prausnitzii," mBio, vol. 6, no. 2, 2015.

[33] T. W. Hoffmann, H.-P. Pham, C. Bridonneau et al., "Microorganisms linked to inflammatory bowel disease-associated dysbiosis differentially impact host physiology in gnotobiotic mice," The ISME Journal, vol. 10, no. 2, pp. 460-477, 2016.

[34] E. Quévrain, M. A. Maubert, C. Michon et al., "Identification of an anti-inflammatory protein from Faecalibacterium prausnitzii, a commensal bacterium deficient in Crohn's disease," Gut, vol. 65, no. 3, pp. 415-425, 2016.
[35] M. Altamimi, O. Abdelhay, and R. A. Rastall, "Effect of oligosaccharides on the adhesion of gut bacteria to human HT29 cells," Anaerobe, vol. 39, pp. 136-142, 2016.

[36] R. M. Jones, "The influence of the gut microbiota on host physiology: in pursuit of mechanisms," The Yale Journal of Biology and Medicine, vol. 89, no. 3, pp. 285-297, 2016.

[37] H. Sokol, B. Pigneur, L. Watterlot et al., "Faecalibacterium prausnitzii is an anti-inflammatory commensal bacterium identified by gut microbiota analysis of Crohn disease patients," Proceedings of the National Academy of Sciences of the United States of America, vol. 105, no. 43, pp. 16731-16736, 2008.

[38] O. Rossi et al., "Faecalibacterium prausnitzii strain HTF-F and its extracellular polymeric matrix attenuate clinical parameters in DSS-induced colitis," PLoS One, vol. 10, no. 4, Article ID e0123013, 2015.

[39] H. Sokol, P. Seksik, L. Rigottier-Gois et al., "Specificities of the fecal microbiota in inflammatory bowel disease," Inflammatory Bowel Diseases, vol. 12, no. 2, pp. 106-111, 2006.

[40] O. Rossi, L. A. van Berkel, F. Chain et al., "Faecalibacterium prausnitzii A2-165 has a high capacity to induce IL-10 in human and murine dendritic cells and modulates $\mathrm{T}$ cell responses," Scientific Reports, vol. 6, no. 1, p. 18507, 2016.

[41] J.-P. Furet, L.-C. Kong, J. Tap et al., "Differential adaptation of human gut microbiota to bariatric surgery-induced weight loss: links with metabolic and low-grade inflammation markers," Diabetes, vol. 59, no. 12, pp. 3049-3057, 2010.

[42] K. R. Groschwitz and S. P. Hogan, "Intestinal barrier function: molecular regulation and disease pathogenesis," Journal of Allergy and Clinical Immunology, vol. 124, no. 1, pp. 3-20, 2009.

[43] A. H. Carlsson, O. Yakymenko, I. Olivier et al., "Faecalibacterium prausnitzii supernatant improves intestinal barrier function in mice DSS colitis," Scandinavian Journal of Gastroenterology, vol. 48, no. 10, pp. 1136-1144, 2013.

[44] R. Martín, S. Miquel, F. Chain et al., "Faecalibacterium prausnitzii prevents physiological damages in a chronic lowgrade inflammation murine model," BMC Microbiology, vol. 15, p. 67, 2015.

[45] L. Laval, R. Martin, J. Natividad et al., "Lactobacillus rhamnosusCNCM I-3690 and the commensal bacteriumFaecalibacterium prausnitziiA2-165 exhibit similar protective effects to induced barrier hyper-permeability in mice," Gut Microbes, vol. 6, no. 1, pp. 1-9, 2015.

[46] A. Wächtershäuser and J. Stein, "Rationale for the luminal provision of butyrate in intestinal diseases," European Journal of Nutrition, vol. 39, no. 4, pp. 164-171, 2000.

[47] S. H. Duncan, G. Holtrop, G. E. Lobley, A. G. Calder, C. S. Stewart, and H. J. Flint, "Contribution of acetate to butyrate formation by human faecal bacteria," British Journal of Nutrition, vol. 91, no. 6, pp. 915-923, 2004.

[48] S. Miquel, R. Martín, O. Rossi et al., "Faecalibacterium prausnitzii and human intestinal health," Current Opinion in Microbiology, vol. 16, no. 3, pp. 255-261, 2013.

[49] G. T. Macfarlane and S. Macfarlane, "Fermentation in the human large intestine," Journal of Clinical Gastroenterology, vol. 45, no. Suppl, pp. S120-S127, 2011.

[50] A. Tiran, H.-J. Gruber, W. F. Graier, A. H. Wagner, E. B. M. van Leeuwen, and B. Tiran, "Aspirin InhibitsChlamydia pneumoniae-induced nuclear factor- $\kappa \mathrm{b}$ activation, cytokine expression, and bacterial development in human endothelial cells," Arteriosclerosis, Thrombosis, and Vascular Biology, vol. 22, no. 7, pp. 1075-1080, 2002. 
[51] H.-N. Liu, H. Wu, Y.-Z. Chen, Y.-J. Chen, X.-Z. Shen, and T.-T. Liu, "Altered molecular signature of intestinal microbiota in irritable bowel syndrome patients compared with healthy controls: a systematic review and meta-analysis," Digestive and Liver Disease, vol. 49, no. 4, pp. 331-337, 2017.

[52] M. Lopez-Siles, M. Martinez-Medina, R. Surís-Valls et al., "Changes in the abundance of Faecalibacterium prausnitzii phylogroups I and II in the intestinal mucosa of inflammatory bowel disease and patients with colorectal cancer," Inflammatory Bowel Diseases, vol. 22, no. 1, pp. 28-41, 2016.

[53] L. Chen, W. Wang, R. Zhou et al., "Characteristics of fecal and mucosa-associated microbiota in Chinese patients with inflammatory bowel disease," Medicine, vol. 93, no. 8, p. e51, 2014.

[54] E. Varela, C. Manichanh, M. Gallart et al., "Colonisation byFaecalibacterium prausnitziiand maintenance of clinical remission in patients with ulcerative colitis," Alimentary Pharmacology \& Therapeutics, vol. 38, no. 2, pp. 151-161, 2013.

[55] K. Machiels, M. Joossens, J. Sabino et al., "A decrease of the butyrate-producing speciesRoseburia hominisand Faecalibacterium prausnitzii defines dysbiosis in patients with ulcerative colitis," Gut, vol. 63, no. 8, pp. 1275-1283, 2014.

[56] H. Zhao, "Systematic review and meta-analysis of the role of Faecalibacterium prausnitzii alteration in inflammatory bowel disease," Journal of Gastroenterology and Hepatology, vol. 36, no. 2, pp. 320-328, 2020.

[57] H. Schäffler, A. Kaschitzki, C. Alberts et al., "Alterations in the mucosa-associated bacterial composition in Crohn's disease: a pilot study," International Journal of Colorectal Disease, vol. 31, no. 5, pp. 961-971, 2016.

[58] B. Vakili, A. Fateh, H. Asadzadeh Aghdaei, F. Sotoodehnejadnematalahi, and S. D. Siadat, "Intestinal microbiota in elderly inpatients with clostridioides difficile infection," Infection and Drug Resistance, vol. 13, pp. 27232731, 2020.

[59] M. Demirci, H. B. Tokman, H. K. Uysal et al., "Reduced Akkermansia muciniphila and Faecalibacterium prausnitzii levels in the gut microbiota of children with allergic asthma," Allergologia et Immunopathologia, vol. 47, no. 4, pp. 365-371, 2019.

[60] S. Roychowdhury, J. Cadnum, B. Glueck, M. Obrenovich, C. Donskey, and G. A. M. Cresci, "Faecalibacterium prausnitzii and a prebiotic protect intestinal health in a mouse model of antibiotic and Clostridium difficile exposure," Journal of Parenteral and Enteral Nutrition, vol. 42, no. 7, pp. 1156-1167, 2018.

[61] Q. Le Bastard, T. Ward, D. Sidiropoulos et al., "Fecal microbiota transplantation reverses antibiotic and chemotherapy-induced gut dysbiosis in mice," Scientific Reports, vol. 8, no. 1, p. 6219, 2018.

[62] A. Cattaneo, N. Cattane, S. Galluzzi et al., "Association of brain amyloidosis with pro-inflammatory gut bacterial taxa and peripheral inflammation markers in cognitively impaired elderly," Neurobiology of Aging, vol. 49, pp. 60-68, 2017.

[63] Y. Benno, K. Endo, H. Miyoshi, T. Okuda, H. Koishi, and T. Mitsuoka, "Effect of rice fiber on human fecal microflora," Microbiology and Immunology, vol. 33, no. 5, pp. 435-440, 1989.

[64] D. G. Burke, "The altered gut microbiota in adults with cystic fibrosis," BMC Microbiology, vol. 17, no. 1, p. 58, 2017.

[65] T. Yang, M. M. Santisteban, V. Rodriguez et al., "Gut dysbiosis is linked to hypertension," Hypertension, vol. 65, no. 6, pp. 1331-1340, 2015.
[66] P. Blatchford, H. Stoklosinski, S. Eady et al., "Consumption of kiwifruit capsules increases Faecalibacterium prausnitzii abundance in functionally constipated individuals: a randomised controlled human trial," Journal of Nutritional Science, vol. 6, p. e52, 2017.

[67] K. Rea, S. M. O’Mahony, T. G. Dinan, and J. F. Cryan, "The role of the gastrointestinal microbiota in visceral pain," Gastrointestinal Pharmacology, vol. 239, pp. 269-287, 2017.

[68] S. Musilova, N. Modrackova, P. Hermanova et al., "Assessment of the synbiotic properites of human milk oligosaccharides and Bifidobacterium longum subsp. infantis in vitro and in humanised mice," Beneficial Microbes, vol. 8, no. 2, pp. 281-289, 2017.

[69] A. Heinken, M. T. Khan, G. Paglia, D. A. Rodionov, H. J. M. Harmsen, and I. Thiele, "Functional metabolic map of Faecalibacterium prausnitzii, a beneficial human gut microbe," Journal of Bacteriology, vol. 196, no. 18, pp. 3289-3302, 2014.

[70] C. McGill, V. Iii 3rd, and L. Devareddy, "Ten-year trends in fiber and whole grain intakes and food sources for the United States population: national health and nutrition examination survey 2001-2010," Nutrients, vol. 7, no. 2, pp. 1119-1130, 2015.

[71] C. Haro, S. Garcia-Carpintero, J. F. Alcala-Diaz et al., “The gut microbial community in metabolic syndrome patients is modified by diet," The Journal of Nutritional Biochemistry, vol. 27, pp. 27-31, 2016.

[72] E. van Nood, A. Vrieze, M. Nieuwdorp et al., "Duodenal infusion of donor feces for RecurrentClostridium difficile," New England Journal of Medicine, vol. 368, no. 5, pp. 407-415, 2013.

[73] B. Cui, P. Li, L. Xu et al., "Step-up fecal microbiota transplantation (FMT) strategy," Gut Microbes, vol. 7, no. 4, pp. 323-328, 2016.

[74] A. Weingarden, "Dynamic changes in short- and long-term bacterial composition following fecal microbiota transplantation for recurrent Clostridium difficile infection," Microbiome, vol. 3, p. 10, 2015.

[75] H. T. Chen, "Fecal microbiota transplantation ameliorates active ulcerative colitis," Experimental and Therapeutic Medicine, vol. 19, no. 4, pp. 2650-2660, 2020.

[76] G. Sarrabayrouse, “Mucosal microbial load in Crohn's disease: a potential predictor of response to faecal microbiota transplantation," EBioMedicine, vol. 51, Article ID 102611, 2020.

[77] V. K. Ridaura, J. J. Faith, F. E. Rey et al., "Gut microbiota from twins discordant for obesity modulate metabolism in mice," Science (New York, N.Y.), vol. 341, no. 6150, Article ID 1241214, 2013.

[78] M. T. Khan, S. H. Duncan, A. J. M. Stams, J. M. van Dijl, H. J. Flint, and H. J. M. Harmsen, "The gut anaerobe Faecalibacterium prausnitzii uses an extracellular electron shuttle to grow at oxic-anoxic interphases," The ISME Journal, vol. 6, no. 8, pp. 1578-1585, 2012.

[79] M. T. Khan, J. M. van Dijl, and H. J. Harmsen, “Antioxidants keep the potentially probiotic but highly oxygen-sensitive human gut bacterium Faecalibacterium prausnitzii alive at ambient air," PLoS One, vol. 9, no. 5, Article ID e96097, 2014.

[80] M. Lopez-Siles, "Mucosa-associated Faecalibacterium prausnitzii and Escherichia coli co-abundance can distinguish irritable bowel syndrome and inflammatory bowel disease phenotypes," International Journal of Medical Microbiology, vol. 304, no. 3-4, pp. 464-475, 2014.

[81] A. Vrieze, E. Van Nood, F. Holleman et al., "Transfer of intestinal microbiota from lean donors increases insulin 
sensitivity in individuals with metabolic syndrome," Gastroenterology, vol. 143, no. 4, pp. 913-916, 2012.

[82] G. Sarrabayrouse, "CD4CD8 $\alpha \alpha$ lymphocytes, a novel human regulatory $\mathrm{T}$ cell subset induced by colonic bacteria and deficient in patients with inflammatory bowel disease," PLoS Biology, vol. 12, no. 4, Article ID e1001833, 2014.

[83] M. Lorea Baroja, P. V. Kirjavainen, S. Hekmat, and G. Reid, "Anti-inflammatory effects of probiotic yogurt in inflammatory bowel disease patients," Clinical \& Experimental Immunology, vol. 149, no. 3, pp. 470-479, 2007.

[84] S. Hanada, M. Pirzadeh, K. Y. Carver, and J. C. Deng, "Respiratory viral infection-induced microbiome alterations and secondary bacterial pneumonia," Front Immunol, vol. 9, p. 2640, 2018.

[85] D. Dhar and A. Mohanty, "Gut microbiota and Covid-19possible link and implications," Virus Research, vol. 285, Article ID 198018, 2020.

[86] F. Anwar, H. N. Altayb, F. A. Al-Abbasi, A. L. Al-Malki, M. A. Kamal, and V. Kumar, "Antiviral effects of probiotic metabolites on COVID-19," Journal of Biomolecular Structure and Dynamics, vol. 2020, pp. 1-10, 2020.

[87] Z. Shen, Y. Xiao, L. Kang et al., "Genomic diversity of severe acute respiratory syndrome-coronavirus 2 in patients with coronavirus disease 2019," Clinical Infectious Diseases, vol. 71, no. 15, pp. 713-720, 2020.

[88] T. Emoto, T. Yamashita, N. Sasaki et al., "Analysis of gut microbiota in coronary artery disease patients: a possible link between gut microbiota and coronary artery disease," Journal of Atherosclerosis and Thrombosis, vol. 23, no. 8, pp. 908-921, 2016.

[89] R. E. Ley, P. J. Turnbaugh, S. Klein, and J. I. Gordon, "Human gut microbes associated with obesity," Nature, vol. 444, no. 7122, pp. 1022-1023, 2006.

[90] J. Shang, G. Ye, K. Shi et al., "Structural basis of receptor recognition by SARS-CoV-2," Nature, vol. 581, no. 7807, pp. 221-224, 2020.

[91] T. Hashimoto, T. Perlot, A. Rehman et al., "ACE2 links amino acid malnutrition to microbial ecology and intestinal inflammation," Nature, vol. 487, no. 7408, pp. 477-481, 2012.

[92] N. Geva-Zatorsky, E. Sefik, L. Kua et al., "Mining the human gut microbiota for immunomodulatory organisms," Cell, vol. 168, no. 5, pp. 928-943, 2017.

[93] T. Zuo, F. Zhang, G. C. Y. Lui et al., "Alterations in gut microbiota of patients with COVID-19 during time of hospitalization," Gastroenterology, vol. 159, no. 3, pp. 944-955, 2020.

[94] Q. Cai, F. Chen, T. Wang et al., "Obesity and COVID-19 severity in a designated hospital in Shenzhen, China," Diabetes Care, vol. 43, no. 7, pp. 1392-1398, 2020.

[95] M. A. Hill, C. Mantzoros, and J. R. Sowers, "Commentary: COVID-19 in patients with diabetes," Metabolism, vol. 107, Article ID 154217, 2020.

[96] K. E. Wellen and G. S. Hotamisligil, "Inflammation, stress, and diabetes," Journal of Clinical Investigation, vol. 115, no. 5, pp. 1111-1119, 2005.

[97] F. J. Verdam, S. Fuentes, C. de Jonge et al., "Human intestinal microbiota composition is associated with local and systemic inflammation in obesity," Obesity, vol. 21, no. 12, pp. E607-E615, 2013.

[98] A. C. de Moraes, G. R. Fernandes, I. T. da Silva et al., "Enterotype may drive the dietary-associated cardiometabolic risk factors," Frontiers in Cellular and Infection Microbiology, vol. 7, p. 47, 2017. 